\title{
Excellent daytime seeing at Dome Fuji on the Antarctic plateau
}

\author{
H. Okita ${ }^{1}$, T. Ichikawa ${ }^{1}$, M. C. B. Ashley ${ }^{2}$, N. Takato ${ }^{3}$, and H. Motoyama ${ }^{4}$ \\ 1 Astronomical Institute, Tohoku University, 6-3 Aramaki, 980-8578 Aoba-ku Sendai, Japan \\ e-mail: h-okita@astr.tohoku.ac.jp \\ 2 School of Physics, University of New South Wales, Sydney, NSW 2052, Australia \\ 3 Subaru Telescope, 650 North A'ohoku Place, Hilo, HI 96720, USA \\ ${ }^{4}$ National Institute of Polar Research, 10-3, Midoricho, Tachikawa, 190-8518 Tokyo, Japan
}

Received 22 May 2013 / Accepted 23 May 2013

ABSTRACT

Context. Dome Fuji, the second highest region on the Antarctic plateau, is expected to have some of the best astronomical seeing on Earth. However, site testing at Dome Fuji is still in its very early stages.

Aims. We investigate the astronomical seeing in the free atmosphere above Dome Fuji and determine the height of the surface boundary layer.

Methods. A Differential Image Motion Monitor was used to measure the seeing in the visible (472 nm) at a height of $11 \mathrm{~m}$ above the snow surface at Dome Fuji during the austral summer of 2012/2013.

Results. Seeing below $0.2^{\prime \prime}$ has been observed. The seeing often has a local minimum of $\sim 0.3^{\prime \prime}$ near $18 \mathrm{~h}$ local time. Some periods of excellent seeing, $0.3^{\prime \prime}$ or smaller, were also observed, sometimes extending for several hours around local midnight. The median seeing is larger, at $0.52^{\prime \prime}$. This high value is believed to be caused by periods when the telescope was within the turbulent boundary layer.

Conclusions. The diurnal variation in the daytime seeing at Dome Fuji is similar to what is reported for Dome C, and the height of the surface boundary layer is consistent with previous simulations for Dome Fuji. The free atmosphere seeing is $\sim 0.2^{\prime \prime}$, and the height of the surface boundary layer can be as low as $\sim 11 \mathrm{~m}$.

Key words. site testing

\section{Introduction}

Dome Fuji is located at $77^{\circ} 19^{\prime} \mathrm{S} 39^{\circ} 42^{\prime} \mathrm{E}$, and with a height of $3810 \mathrm{~m}$, it is the second highest region on the Antarctic plateau. Astronomical seeing is generally considered as the superposition of the contributions from two layers: the surface boundary layer and the free atmosphere above. Measurements at Dome C, which is another high region on the Antarctic plateau, have shown the best seeing so far observed from the Earth with a free atmosphere seeing of $\sim 0.3^{\prime \prime}$ and a surface boundary layer thickness of $\sim 30 \mathrm{~m}$ (Lawrence et al. 2004; Aristidi et al. 2009). At Dome A, the highest region on the Antarctic plateau, the median height of the surface boundary layer has been measured as $\sim 14 \mathrm{~m}$ (Bonner et al. 2010). Simulations suggest that the free atmosphere seeing above Dome Fuji and the height of the surface boundary layer could be 0.21" and $18 \mathrm{~m}$ (Saunders et al. 2009; Swain \& Gallée 2006). We conducted a site-testing campaign over the 2012/2013 summer in an attempt to test these expectations.

\section{Instrumentation}

The Differential Image Motion Monitor (DIMM) is a commonly used instrument to measure the seeing. The DIMM works by using two subapertures on a small telescope, with a wedge prism attached, to make two images of the same star on a CCD detector. The seeing can be calculated by measuring the differential motion between these two images and assuming Kolmogorov turbulence (Sarazin \& Roddier 1990).

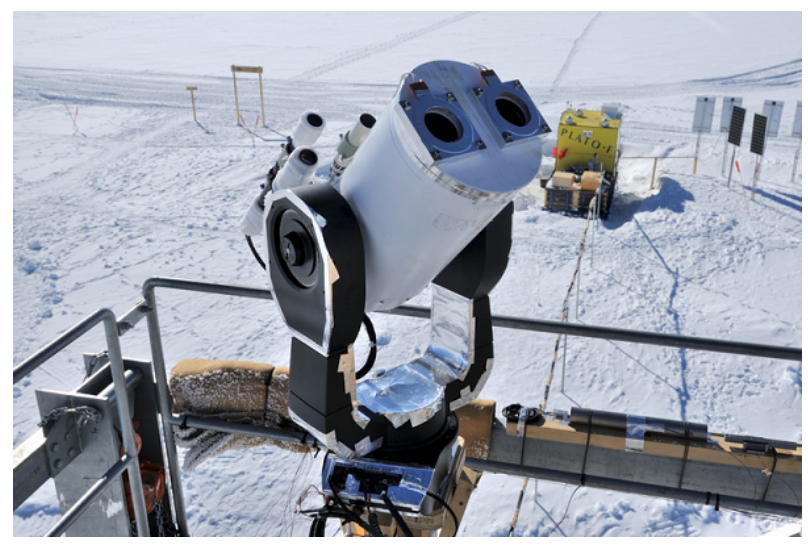

Fig. 1. DF-DIMM on the $9 \mathrm{~m}$ tower at Dome Fuji, 2013 January. The telescope aperture is $\sim 11 \mathrm{~m}$ above the snow surface.

Our instrument, the "Dome Fuji Differential Image Motion Monitor" (DF-DIMM), is based on a Meade LX200-8" Cassegrain telescope, with an SBIG ST-i monochrome CCD camera equipped with an Edmund narrow-band filter at $472 \mathrm{~nm}$ for suppressing auroral emissions (Okita et al. 2013). DF-DIMM (see Fig. 1) was operated fully automatically to allow the efficient accumulation of many seeing estimates. The optical tube of the telescope was painted white to minimize the local turbulence inside and around the tube generated by the solar radiation. Many modifications were made to allow operation in the 
Table 1. Parameters and technical specifications of DF-DIMM.

\begin{tabular}{lc}
\hline \hline Subaperture diameter & $\phi 60 \mathrm{~mm}$ \\
Subaperture separation & $140 \mathrm{~mm}$ \\
Observed wavelength & $472 \mathrm{~nm}$ \\
FWHM of the filter & $35 \mathrm{~nm}$ \\
Pixel size & $7.4 \mu \mathrm{m} \times 7.4 \mu \mathrm{m}$ \\
Pixel scale & $0.775^{\prime \prime} / \mathrm{pix} \pm 0.005^{\prime \prime} / \mathrm{pix}$ \\
Exposure time & $0.001 \mathrm{~s}$ \\
Number of frames used & \\
$\quad$ for each seeing estimate & 450 over $\sim 5 \mathrm{~min}$ \\
Height of the entrance pupils & $\sim 11 \mathrm{~m}$ \\
\hline
\end{tabular}

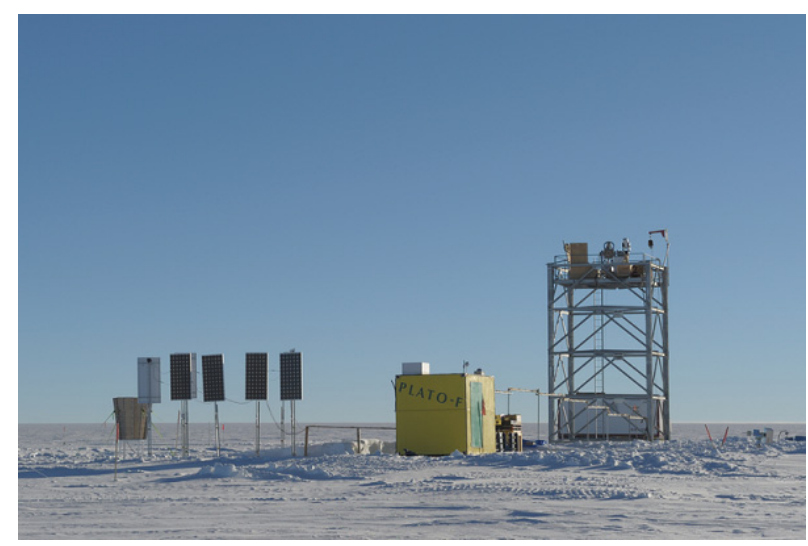

Fig. 2. PLATO-F (center, yellow container) and the $9 \mathrm{~m}$ tower (right) with DF-DIMM on top. The rectangular objects to the left are solar panels.

Antarctic environment. Table 1 summarizes the parameters and the technical specifications of DF-DIMM.

DF-DIMM observed Canopus ( $\alpha$ Car, $V=-0.7 \mathrm{mag}$, the second brightest star in the sky) to measure the seeing. Canopus is circumpolar at Dome Fuji, with a zenith angle varying from $25^{\circ}$ to $50^{\circ}$. DF-DIMM could observe Canopus continuously for days at a time with a reasonable contrast against the daytime sky background.

DF-DIMM was placed on the top of a $9 \mathrm{~m}$ tower in order to be as high as possible within, and sometimes above, the surface boundary layer. The height of the entrance pupils of DF-DIMM was $\sim 11 \mathrm{~m}$.

DF-DIMM was supported by PLATO-F (PLATeau Observatory for Dome Fuji), a fully automated observing platform for the Antarctic plateau deployed at Dome Fuji in January 2011. Figure 2 shows PLATO-F and the $9 \mathrm{~m}$ tower. PLATO-F provides electrical power of up to $1 \mathrm{~kW}$ and Iridium communications all year for site testings and astronomical observations from Dome Fuji (Ashley et al. 2010).

\section{Data processing and error analysis}

On-site data processing software was developed for DF-DIMM, based on Nightview ${ }^{1}$ (Hroch), Sextractor (Bertin \& Arnouts 1996), and CFITSIO (Pence 1999). The longitudinal seeing $\epsilon_{1}$ and the transverse seeing $\epsilon_{\mathrm{t}}$ were calculated using the Eqs. (13), (14), and (23) of Sarazin \& Roddier (1990) from 450 images taken at about five-minute intervals. The seeing estimates were then corrected for zenith angle using Eq. (24) of their paper.

\footnotetext{
1 http://www.physics.muni.cz/mb/nightview/nightview . html
}

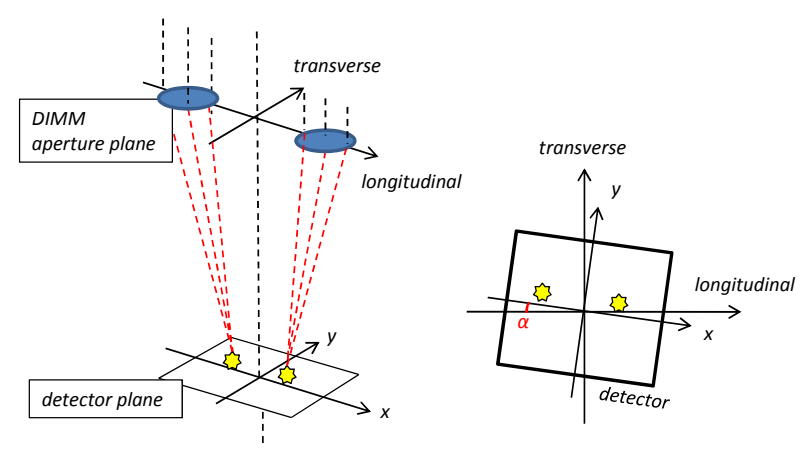

Fig. 3. Schematic showing the ray trace for a generic DIMM. Two subapertures with wedge prisms make two images of the same star on a CCD detector.

The seeing values measured by a DIMM, $\epsilon_{\mathrm{l}}$ and $\epsilon_{\mathrm{t}}$, have statistical error and pixel scale uncertainty. The statistical error of the variance of a star position $\sigma_{\star}^{2}$ is $\mathrm{d} \sigma_{\star}^{2} / \sigma_{\star}^{2}=\sqrt{2 /(N-1)}$, where $N$ is the number of frames used for the variance calculation and the subscript $\star$ represents either longitudinal or transverse (Sarazin \& Roddier 1990). In our case, 450 frames were used in calculating each seeing estimate. As a result, the statistical error on the variance is $\sim 6.7 \%$, which corresponds to a seeing error of $\mathrm{d} \epsilon_{\star} / \epsilon_{\star} \propto\left(\mathrm{d} \sigma_{\star}^{2} / \sigma_{\star}^{2}\right)^{3 / 5} \sim 4 \%$. The pixel scale of DF-DIMM was measured using the diurnal motion of Canopus on January 1. This gives a scale of $0.775^{\prime \prime} \pm 0.005^{\prime \prime}$ per pixel. The uncertainty contributes $\sim 0.8 \%$ error in the seeing. The temperature dependence of the focal length also affects the pixel scale; however this effect is negligibly small for our Cassegrain telescope. In fact, optical simulations demonstrate that the focal length of DF-DIMM changes less than $0.2 \%$ between $20{ }^{\circ} \mathrm{C}$ and $-80{ }^{\circ} \mathrm{C}$.

Seeing should be the same in the longitudinal and transverse directions since seeing is a scalar quantity. Considering the statistical error and pixel scale uncertainty, we discarded $\sim 1 \%$ of observations that fell outside the range $0.50<\epsilon_{\mathrm{l}} / \epsilon_{\mathrm{t}}<2.0$. We then averaged $\epsilon_{1}$ and $\epsilon_{\mathrm{t}}$ to obtain the seeing value.

Other sources of uncertainty in the DIMM measurements are considered below for better estimation of seeing.

\subsection{Instrument rotation effect}

To simplify the analysis of DIMM data, it is usual to align the $(x, y)$ coordinates of the CCD detector with the longitudinal and transverse DIMM coordinates $(l, t)$ defined by Sarazin $\&$ Roddier (1990), see Fig. 3. If, however, these coordinate frames are misaligned by some angle $\alpha$, and this is not corrected for in the analysis, an error will result.

Here we write $\sigma_{x}^{2}$ and $\sigma_{y}^{2}$ as the variance of the differential motion along the $x$ and $y$ axes, and $\sigma_{x y}$ as the covariance of $x$ and $y$. The longitudinal variances of $\sigma_{1}^{2}$ and transverse variance of $\sigma_{t}^{2}$ are then

$\sigma_{1}^{2}=\cos ^{2}(\alpha) \sigma_{x}^{2}+\sin ^{2}(\alpha) \sigma_{y}^{2}-\sin (2 \alpha) \sigma_{x y}$
$\sigma_{t}^{2}=\sin ^{2}(\alpha) \sigma_{x}^{2}+\cos ^{2}(\alpha) \sigma_{y}^{2}+\sin (2 \alpha) \sigma_{x y}$.

For precise measurements of the seeing from DIMM observations we need to transform the $(x, y)$ coordinates to $(l, t)$ before using the normal DIMM equations. 


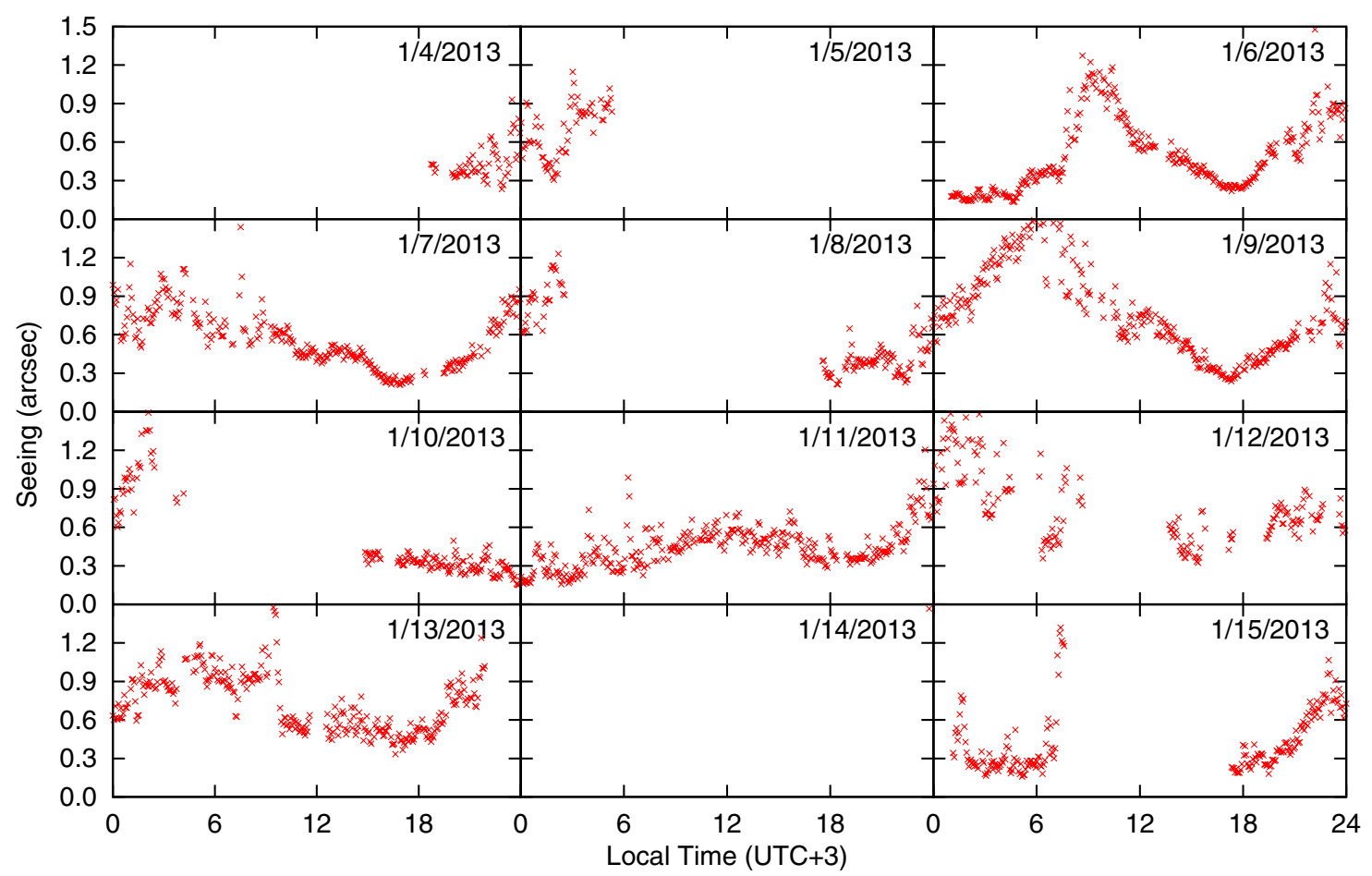

Fig. 4. Time series of Dome Fuji seeing from 2013 January 4 to 15 . The seeing was measured at wavelength of $472 \mathrm{~nm}$ at a height of $11 \mathrm{~m}$ above the snow surface. We plot the average of the longitudinal and transverse seeings.

\subsection{Finite exposure effect}

Theoretically, DIMM seeing is defined in an infinitely short exposure. Martin (1987) and Soules et al. (1996) discussed the effect of using a finite exposure time. From equation (18) of Soules et al. (1996) with $\tau=0.001 \mathrm{~s}$ and $w \leq 30 \mathrm{~m} / \mathrm{s}$, our seeing values are underestimated by less than $3 \%$.

\subsection{Readout, background, and local turbulence effects}

Readout noise and background noise of the detector also add small biases to the seeing value (Tokovinin 2002). The local turbulence inside the telescope worsens the observed seeing. These effects all cause our results to be an upper limit on the actual seeing.

\section{Results}

We carried out DIMM observations $11 \mathrm{~m}$ above the snow surface at a wavelength of $472 \mathrm{~nm}$ from 2013 January 4 to January 23. In all, we obtained 3814 seeing estimates, each one calculated from 450 images over a period of about five-minutes. Figures 4 and 5 show the time series of the seeing, day by day.

A period of excellent seeing, below $0.2^{\prime \prime}$ and continuing for about four hours, was observed near local midnight on 2013 January 6. Other periods of excellent seeing, less than $0.3^{\prime \prime}$, were observed close to local midnight on a total of six occasions (January 6, 11, 15, 19, 21, and 23).

The seeing has a tendency to have a local minimum of $\sim 0.3^{\prime \prime}$ near $18 \mathrm{~h}$ local time. This is clear in the data for January $6,7,9$, and 16

The histogram of the seeing measurements is plotted in Fig. 6. The mean, median, and mode of the seeing values were $0.68^{\prime \prime}, 0.52^{\prime \prime}$, and $0.36^{\prime \prime}$, respectively. The 25 th and the 75 th percentile of seeing were $0.36^{\prime \prime}$ and $0.78^{\prime \prime}$. As discussed below, we expect the higher seeing measurements to be due to periods when the surface boundary layer was above the level of the top of the telescope.

\section{Discussion and conclusion}

We note that the Dome Fuji seeing tends to have its lowest values a few hours around local dusk and midnight. It is remarkable that seeing in the range $0.2^{\prime \prime}$ to $0.3^{\prime \prime}$ was observed for continuous periods of hours at a height of only $11 \mathrm{~m}$ above the snow surface, presumably due to periods where the surface boundary layer is either below the height of the DIMM aperture or has disappeared altogether.

A similar local minimum at local dusk has also been seen at Dome C and been interpreted by Aristidi et al. (2005) as due to the disappearance of the surface boundary layer. Our results are consistent with this.

However, it is interesting to note that the excellent seeing we have observed at local midnight has not been reported from site testing of $8 \mathrm{~m}$ above the snow surface at Dome C. The weak insolation at midnight is expected to result in an intense temperature gradient near the snow surface at this time. This strong temperature gradient should produce a strong surface boundary layer, so there is poor seeing from the surface. This is only consistent with our observations if the surface boundary layer is below the level of our telescope. We therefore conclude that our DIMM was above the surface boundary layer during these periods and was sampling the free atmosphere seeing. A low surface boundary layer has been predicted from simulations by Swain \& Gallée (2006) and is consistent with our observations. Observations with a sonic radar at Dome A have shown that the surface boundary layer is often highly turbulent, but confined to a very thin (14 m median) layer near the snow (Bonner et al. 2010). 


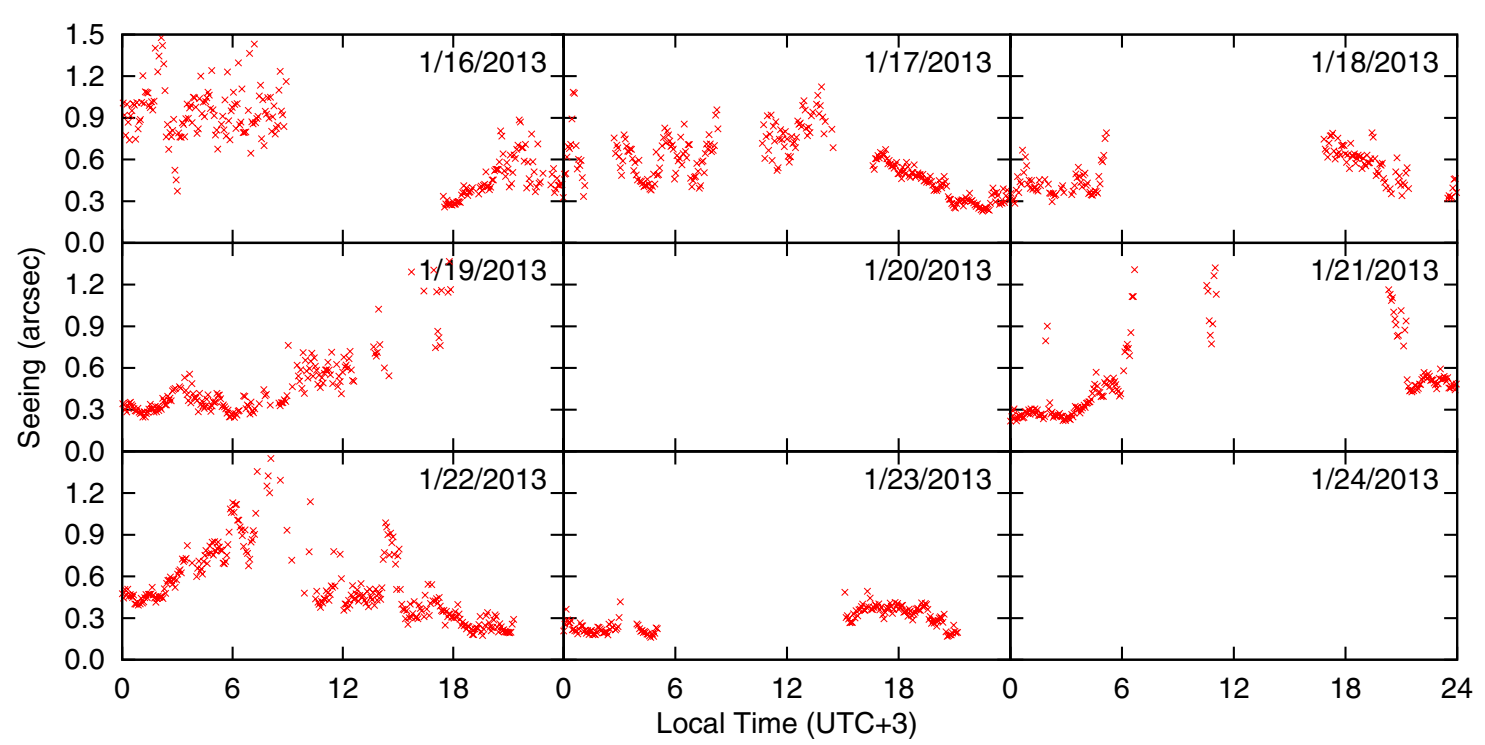

Fig. 5. Same as Fig. 4, but for the period 2013 January 16 to 24 .

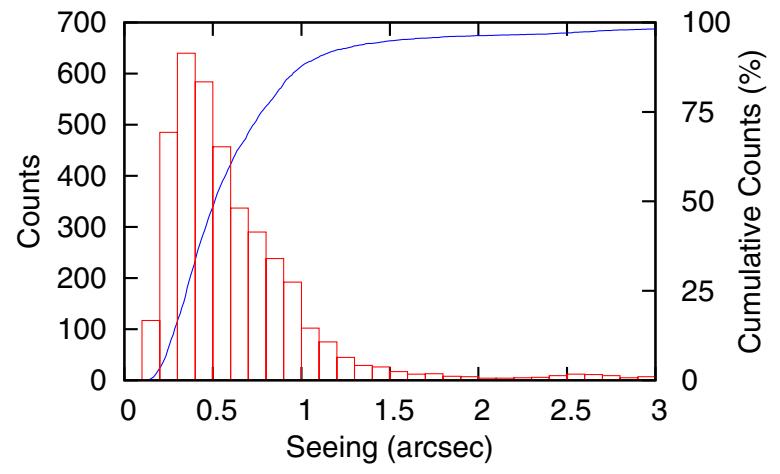

Fig. 6. Histogram (bars) and cumulative histogram (solid line) of Dome Fuji seeing measured from 2013 January 4 to 23. Measurements above $\sim 0.4^{\prime \prime}$ are probably the result of the DIMM being within the surface boundary layer.

The histogram of seeing measurements in Fig. 6 is expected to consist of two sets of data: those when the telescope is outside the surface boundary layer, and those when the telescope is inside. The latter measurements will produce the long tail of seeing measurements above $\sim 0.4^{\prime \prime}$. We expect that if the DIMM was mounted on a higher tower, the fraction of measurements in this tail would drop significantly. A quantitative estimate of this effect could be made from sonic radar measurements of the boundary layer height.

In summary, at a given height above the snow, excellent seeing at Dome Fuji occurs when there is either a low or nonexistent surface boundary layer. The free atmosphere seeing is $\sim 0.2^{\prime \prime}$. The height of the surface boundary layer has been observed to be as low as $\sim 11 \mathrm{~m}$.
Our findings give strong encouragement to constructing future large-aperture telescopes on the Antarctic plateau to take advantage of the excellent natural seeing and the low surface boundary layer. We are now preparing to make wintertime seeing measurements with DF-DIMM.

Acknowledgements. We acknowledge the National Institute of Polar Research and the 51st-54th Japanese Antarctic Research Expeditions. This research is supported by the National Institute of Polar Research through Project Research No. KP-12, the Grants-in-Aid for Scientific Research 18340050 and 23103002, the Australian Research Council and Australian government infrastructure funding managed by Astronomy Australia Limited. Hirofumi Okita thanks the Sasakawa Scientific Research Grant from The Japan Science Society, and Tohoku University International Advanced Research and Education Organization for scholarships and research expenses.

\section{References}

Aristidi, E., Agabi, A., Fossat, E., et al. 2005, A\&A, 444, 651 Aristidi, E., Fossat, E., Agabi, A., et al. 2009, A\&A, 499, 955 Ashley, M. C. B., Bonner, C. S., Everett, J. R., et al. 2010, in SPIE Conf. Ser., 7735

Bertin, E., \& Arnouts, S. 1996, A\&AS, 117, 393

Bonner, C. S., Ashley, M. C. B., Cui, X., et al. 2010, PASP, 122, 1122

Lawrence, J. S., Ashley, M. C. B., Tokovinin, A., \& Travouillon, T. 2004, Nature, 431, 278

Martin, H. M. 1987, PASP, 99, 1360

Okita, H., Takato, N., Ichikawa, T., et al. 2013, in IAU Symp., 288, 25

Pence, W. 1999, in ASP Conf. Ser., Astronomical Data Analysis Software and Systems VIII, eds. D. M. Mehringer, R. L. Plante, \& D. A. Roberts, 172, 487 Sarazin, M., \& Roddier, F. 1990, A\&A, 227, 294

Saunders, W., Lawrence, J. S., Storey, J. W. V., et al. 2009, PASP, 121, 976

Soules, D. B., Drexler, J. J., Draayer, B. F., Eaton, F. D., \& Hines, J. R. 1996, PASP, 108, 817

Swain, M. R., \& Gallée, H. 2006, PASP, 118, 1190

Tokovinin, A. 2002, PASP, 114, 1156 